\title{
Speech changes in patients with a full rehabilitation for severe tooth wear, a first evaluation study
}

\author{
Bernadette A.M.M. Sterenborg ${ }^{1}$ - Stanimira I. Kalaykova ${ }^{1}$ - Simone Knuijt ${ }^{2}$ - Bas A.C. Loomans ${ }^{1}$ • \\ Marie-Charlotte D. N. J. M. Huysmans ${ }^{1}$
}

Received: 17 December 2018 / Accepted: 11 December 2019 / Published online: 19 December 2019

(C) The Author(s) 2019

\begin{abstract}
Objective The aim of this study was to evaluate changes in speech characteristics and self-perceived quality of speech in tooth wear patients, after occlusal rehabilitation.

Materials and methods Patients with tooth wear were included in this study after informed consent. The amount of tooth wear was scored with Tooth Wear Evaluation System (TWES). To assess the perspective of the patient, the Dutch Speech Handicap Index was used (SHI). Acoustic analysis was performed to evaluate changes with the use of voice recordings. These were made before treatment, $T_{0}$; directly after treatment, $T_{1} ; 1$ month after treatment, $T_{2}$. With the use of PRAAT software, the spectral characteristic centre of gravity (COG) was evaluated for the sounds $/ \mathrm{s} /, / \mathrm{f} /, / \mathrm{v} /, / \mathrm{d} /, / \mathrm{t} /, / \mathrm{m} /$.

Results Recordings of 17 patients (14 men, 3 women, mean age $41.2 \pm 10.4$ years) were included. SHI scores did not change significantly between $T_{0}$ and $T_{2}(p=0.054)$. A multiple regression model showed that for all sounds the intercept was negative, but statistically significant only for /s/ and /f/ between $T_{0}$ and $T_{1}$. The effect of the initial change (between $T_{0}$ and $T_{1}$ ) on the change between $T_{1}$ and $T_{2}$ was clearly negative for all sounds $(p<0.001$ ), showing a rebound effect ranging between 29 and $68 \%$ of the initial change.

Conclusion Tooth wear patients perceive improvement in speech function after treatment.

Clinical significance Clinicians may explain to patients that speech is likely to alter for a short period due to treatment but that there will be a good adaption to the new situation.
\end{abstract}

Keywords Tooth wear $\cdot$ Speech articulation $\cdot$ Speech acoustics $\cdot$ Mouth rehabilitation

\section{Introduction}

Tooth wear and its management present new challenges in dentistry [1]. Tooth wear is increasingly common, especially among children and adolescents $[2,3]$. The loss of occlusal tooth substance in tooth wear may result in a reduced vertical dimension of occlusion (VDO) and altered shape of teeth. Patients may need complex restorative treatment such as a full

Bernadette A.M.M. Sterenborg

Bernadette.Sterenborg@radboudumc.nl

1 Radboud Institute for Health Sciences, Department of Preventive and Restorative Dentistry, Radboud University Medical Center, P.O. Box 9101 NL, 6500, HB Nijmegen, The Netherlands

2 Donders Institute for Brain, Cognition and Behaviour, Department of Rehabilitation/Speech therapy, Radboud University Medical Center, Nijmegen, The Netherlands rehabilitation at an increased VDO [4-7]. Changes in tooth shape, both due to wear and its restorative treatment, may have an effect on speech.

Speech is the ability to communicate through articulated sounds. The production of speech is divided into five levels: (1) Respiration, the air flow that is generated by the lungs; (2) Phonation, the production of sounds by the vocal cords; (3) Resonance, vibrations created by the pharynx, the oral and the nasal cavity; (4) Articulation, the formation of sounds by movements of the articulators and (5) Prosody, suprasegmental characteristics like rhythm, intonation and pitch [8]. Tooth abnormalities or missing teeth are most likely to have an effect on articulation. Along with the tongue, the teeth are, for example, involved in the production of $/ \mathrm{f} /$ and $/ \mathrm{v} /$ and help to produce the frication in sounds like $/ \mathrm{s} /$ and $/ \mathrm{z} /$ as the breath stream passes over the lower edges of the incisor teeth [9]. Speech sounds which are thought to be most frequently misarticulated after an increase in VDO include /s/ and /sh/, 
sounds which are produced when exhaled air enters the mouth and is guided by the tongue [10]. Although previous studies have investigated the effect of tooth wear on oral functions [11-13], there is to our knowledge little to no research about the effect on speech of a full rehabilitation of the worn dentition with an increase in VDO.

To evaluate speech sounds, the parameter centre of gravity (COG) may be used. This objective parameter quantifies a spectral detail that corresponds to the frequency of the speech sound [14]. A subjective evaluation of speech characteristics as experienced by patients with tooth wear is possible with the use of a questionnaire, in this case the Dutch Speech Handicap Index (SHI) [15].

The aim of this first evaluation study was to explore changes in speech characteristics and self-perceived quality of speech in tooth wear patients, after a full rehabilitation with an increase in VDO. A second aim was to evaluate the possible adaptation in speech characteristics and self-perceived quality of speech at follow-up.

\section{Materials and methods}

\section{Patients}

Patients with tooth wear referred by general dental practitioners to the Department of Dentistry of the Radboud University Medical Center (Nijmegen, The Netherlands) were eligible for inclusion. The inclusion took place in the period January 2015 till January 2018. Ethical approval was granted before study commencement (NL2014-1252). All patients who were asked to participate agreed and signed an informed consent before entering the study.

\section{Inclusion/exclusion criteria}

The following inclusion criteria were applied: (1) age $\geq$ 18 years, (2) generalized tooth wear with need for a full rehabilitation, (3) full dental arches, with a maximum of one missing tooth in the posterior area, (4) absence of general health problems (ASA-score $\leq 3$ ) and (5) Dutch native speaker.

The following exclusion criteria were used: (1) mouth opening $<3.5 \mathrm{~cm}$, (2) temporomandibular disorders, (3) periodontitis (pockets $>4 \mathrm{~mm}$ ) and (4) acute dental caries or endodontic problems.

\section{Tooth Wear Evaluation System}

The Tooth Wear Evaluation System (TWES) is a modular clinical guideline, which aims at optimising the diagnostic process (both qualifying and quantifying) and improving the selection of proper management strategies regarding tooth wear for the permanent dentition [16]. TWES was evaluated on intraoral 3D scans of the patients' dentitions. For occlusal and incisal grading an eight-point ordinal scale was used. A three-point ordinal scale for non-occlusal/non-incisal grading was used.

\section{VDO increase and lengthening of upper incisors}

To determine the applied VDO and the difference in length of the upper incisors after treatment, intraoral 3D scans, made before and after treatment, were used. Meshlab (http:// meshlab.sourceforge.net/) was used to evaluate VDO increase (the difference in height measured at the first molars) and incisal lengthening of the upper central incisors due to the full rehabilitation.

\section{Full rehabilitation of tooth wear}

Based on initial examination, an individual treatment plan was made for each patient [4]. The required VDO increase was estimated taking into account the amount of tooth wear at the first molars and the need or possibility to lengthen the anterior teeth. The optimal length of the anterior teeth was determined with an intraoral mock-up in the upper jaw from cuspid to cuspid with direct composite restorations, using the lip-generated smile design [17]. The increase usually lies between 2 and $4 \mathrm{~mm}$ in the first molar region. Based on the digital scan and intra-oral mock-up, a digital wax-up was made by a dental technician, and indirect composite restorations (LAVA Ultimate, $3 \mathrm{M}^{\mathrm{TM}}$ ) were milled from a prepolymerized block [4]. Restorations were placed in multiple sessions, and a final appointment was made to make direct composite veneers (Filtek Supreme XTE, $3 \mathrm{M}^{\mathrm{TM}}$ ) on the upper and lower anterior teeth.

\section{Self-evaluation of speech}

To assess the patients' perspective regarding his/her speech, the Dutch Speech Handicap Index (SHI) [15] was used on three occasions: before treatment $\left(T_{0}\right)$, immediately after treatment $\left(T_{1}\right)$ and 1 month after treatment $\left(T_{2}\right)$. SHI is a selfevaluation questionnaire originally designed and validated to measure the physical, functional and emotional impact on speech due to dysarthria. Since there is no questionnaire available to evaluate speech in relation to tooth wear, SHI was considered the best available option. This questionnaire consisted of two parts. Part 1 contains 15 items, split into three subscales of 5 items each: physical, functional and emotional functions. Each item is scored on a five-point scale (never (0), almost never (1), sometimes (2), almost always (3) and always (4)). The maximum score per subscale was 20; the total overall score was 60 . Finally, all answers were summed to obtain an overall score. The second part consists of 3 general questions on which the patient could choose a numeric rating score 
(NRS) between 0 to 10 , meaning 0 no impact, 10 meaning maximal impact. These questions are a summary of the previous questions, but the scoring scale is different. The scores of part 2 are not used in the total score but give an extra impression of the experience of the patient. In the analysis in this study, only part 1 was used for clear interpretation; part 2 was excluded from analysis.

\section{Speech recordings}

Recordings of the patients' speech on the same three occasions: before treatment $\left(T_{0}\right)$, immediately after treatment $\left(T_{1}\right)$ and 1 month after treatment $\left(T_{2}\right)$. The recorded material consisted of the Dutch text 'De Koning' [18]. All speech samples were taken with the same Tascam DR-05 recording machine, under standard conditions in a sound isolated room at the audiological center of the Radboud University Medical Center. The recording at $T_{1}$ took place immediately after the final treatment session, in which no anaesthesia (possibly interfering with speech) was required. The final session took place approximately 1 month after treatment $\left(T_{2}\right)$. After every speech recording, the patients filled out the SHI.

\section{Acoustic analysis}

The speech sounds evaluated were the $/ \mathrm{s} /, / \mathrm{f} /, / \mathrm{v} /, \mathrm{d} /, / \mathrm{t} /, / \mathrm{m} /$. The sounds located at the beginning of words were analysed because this position makes it easier and more reliable to isolate them for acoustic analysis. In the text 'De Koning', the $/ \mathrm{s} /, / \mathrm{v} /, / \mathrm{d} /, / \mathrm{t} /, / \mathrm{m} /$ occur 5 times at the beginning of a word, the /f/ 4 times. Using PRAAT software (version 6.0.43) [14], digital samples of the sounds were made. The signals were sampled at $44.1 \mathrm{kHz}$. The cursor was placed manually at the start and end of each sound using a Hamming window. A PRAAT script was developed to derive the spectral characteristic centre of gravity (COG) expressed in $\mathrm{Hz}$, which meant that the highest value in $\mathrm{Hz}$ was taken into account for the analysis. The value of the COG was measured for every appointed sound in the three different recordings. The use of a filter or a pre-emphasis was not deemed necessary because all recordings took place in a sound isolated room.

\section{Statistical analysis}

Paired Student's $t$ test was used to calculate intra-observer reproducibility of the acoustic analysis. Reliability coefficient was calculated as Pearson's correlation coefficient through analysing $10 \%$ of the recordings twice. The duplicate measurement error was calculated as the standard deviation of the differences between round 1 and 2 , divided by $\sqrt{ } 2$. To analyse a possible non-random difference between repeated measurements, the mean difference $(95 \% \mathrm{CI}, p<0.05)$ was calculated.
SHI scores were analysed with paired $t$ tests to analyse the overall effect of the treatment and 1 month of adaptation (i.e. comparison between outcomes at $T_{0}-T_{2}$ ). To evaluate each question separately, a Wilcoxon signed rank test was used. For all six sounds, the change of COG in the period directly after treatment was analysed using a multiple regression model. As we have multiple observations (4 or 5) within one sound for each patient, a multilevel model was used with a random intercept to allow for the presence of data clustered in patients. To this model, the increase in length of the 2 middle incisors of the upper jaw was added as independent variable. For ease of interpretation, this value was centered around its mean $(2.6 \mathrm{~mm})$. Also, for all six sounds, the effect of adaptation on COG $\left(T_{2}-T_{1}\right)$ was analysed using a multiple regression model, taken into account the amount of change in COG for the period $T_{1}-T_{0}$ as independent variable. All statistical analyses were performed with the Statistical package for Social Sciences (SPSS v.24) or R (v.3.4.3).

\section{Results}

In total, 24 patients were included in the study. Four patients were not able to go the audiological center due to mobility problems (e.g. wheelchair user), and for three patients recordings could not be used due to poor quality of at least one of the recordings. Therefore, the recordings of 17 patients were included: 14 men and 3 women with a mean age of $41.2 \pm$ 10.4 years. TWES scores are shown in Table 1. For the occlusal/incisal wear, the highest score was $3 \mathrm{a}$ in 5 patients, $3 \mathrm{~b}$ for 11 patients and 4 for 1 patient. The mean VDO increase was $2.7 \pm 0.73 \mathrm{~mm}$. The mean lengthening of the central incisors of the upper jaw was $2.6 \pm 1.2 \mathrm{~mm}$. The time interval between treatment and 1-month evaluation $\left(T_{1}\right.$ to $\left.T_{2}\right)$ was on average $42 \pm 17$ days.

The results of the intra-observer reproducibility analysis for the acoustic analysis are shown in Table 2. The $p$ values of the reliability scores are all $>0.98$, indicating no statistically significant differences. Only the sound $/ \mathrm{m} /$ showed a significant $\mathrm{p}$ value of 0.04 .

In Table 3, results of the paired $t$ test analysis of the SHI scores were given for $T_{0}-T_{2}$. The overall score between $T_{0}$ and $T_{2}$ did not change significantly, $p=0.054$, but had a trend for positive improvement, as the overall score was lower at $T_{2}$. One subscale, functional, showed a significant change, $p=$ 0.001 . The other subscales, physical and emotional, showed no significant difference, but for all three subscales individually, the trend was for a positive improvement in speech after treatment. To evaluate the change per question, a Wilcoxon signed rank test was used. Three questions stood out for this specific study group; P2: 'I experience difficulties in articulation when I speak'; F3: 'I find it difficult to communicate with people I don't know' and F4: 'Because of my speech problem 
Table 1 Distribution of overall occlusal/incisal and nonocclusal/nonincisal tooth wear scores, measured on 3D scans. Not all patients were fully dentate; $2.7 \%$ of the teeth were missing

\begin{tabular}{|c|c|c|c|c|c|c|c|c|c|c|c|c|c|}
\hline \multirow[b]{3}{*}{0} & \multicolumn{7}{|c|}{ Occlusal/incisal (\%) } & & \multicolumn{5}{|c|}{ Nonocclusal/nonincisal (\%) } \\
\hline & \multirow[b]{2}{*}{$1 \mathrm{a}$} & \multirow[b]{2}{*}{$1 b$} & \multirow[b]{2}{*}{$1 \mathrm{c}$} & \multirow[b]{2}{*}{2} & \multirow[b]{2}{*}{$3 a$} & \multirow[b]{2}{*}{$3 b$} & \multirow[b]{2}{*}{4} & \multicolumn{3}{|c|}{ Vestibular } & \multicolumn{3}{|c|}{ Lingual } \\
\hline & & & & & & & & 0 & 1 & 2 & 0 & 1 & 2 \\
\hline 0.0 & 0.2 & 0.0 & 2.5 & 39.9 & 33.2 & 19.5 & 1.7 & 0.8 & 7.1 & 89.3 & 1.7 & 12.6 & 83.0 \\
\hline
\end{tabular}

people ask me often to repeat myself'. These questions showed a significant positive difference, resp. 0.021, 0.008 and 0.015 . Table 4 summarizes the change in COG over time. For all sounds, the intercept was negative (signifying a mean drop in COG), but this was only statistically significant for $/ \mathrm{s} /$ and /f/. The effect of the extent of incisor lengthening varied. For the sounds /t/ and /f/ that effect was statistically significant, with a larger change in incisor length being associated with an increase in COG. For the adaptation effect $\left(T_{1}-T_{2}\right)$, the intercept in the analysis does not have a natural interpretation, but the effect of the initial change (between $T_{0}$ and $T_{1}$ ) on the change between $T_{1}$ and $T_{2}$ was clearly negative for all sounds, with always $p<0.001$. That implies that for all sounds the change between $T_{1}$ and $T_{2}$ can be seen as a rebound to the initial change of the COG. Figure 1 visualizes this rebound effect for the sound $/ \mathrm{v} /$. The size of the rebound varied between 29 and $68 \%$ of the initial change in COG (Table 4).

\section{Discussion}

To our knowledge, this is a first evaluation study to investigate the effect of a full occlusal rehabilitation of tooth wear with increase in VDO on speech characteristics. No power analysis was performed on forehand but the results showed, for the majority, a very low $p$ value.

In collaboration with a speech-language pathologist, it was decided to evaluate the sounds most likely to be affected by the increase in vertical dimension or the change in shape of the upper incisors. The intraoral areas that are involved into

Table 2 Results of the intra-observer analysis with the program PRAAT

\begin{tabular}{llrllll}
\hline \multirow{2}{*}{ Sounds } & Reliability & DME & \multicolumn{4}{l}{ Structural difference } \\
\cline { 4 - 6 } & & & Diff. & $95 \%$ CI diff & $p$ value \\
\hline /s/ & 0.996 & 95.49 & 16.72 & {$[-39.02-72.46]$} & 0.542 \\
/t/ & 0.989 & 249.83 & 53.52 & {$[-92.31-199.36]$} & 0.456 \\
/d/ & 0.983 & 127.67 & 17.64 & {$[-56.89-92.17]$} & 0.630 \\
/f/ & 0.989 & 326.93 & 38.65 & {$[-177.74-255.04]$} & 0.713 \\
/v/ & 0.985 & 270.80 & 52.44 & {$[-105.64-210.52]$} & 0.500 \\
/m/ & 0.984 & 7.47 & 4.60 & {$[0.24-8.96]$} & 0.040 \\
\hline
\end{tabular}

articulation of the different sounds in the Dutch language has previously been described by Rietveld et al. [19]. Another decision made based on the expertise of the speechlanguage pathologist, due to the lack of evidence in the literature, was to evaluate the sounds at the beginning of words. This decision was made to make it easier and more reliable to isolate the sounds using the program PRAAT. Intra-observer reliability rates were very high: $>0.98$. Only for the sound $/ \mathrm{m} /$ the re-measurement showed a significant inconsistency with a $p$ value of 0.04 . The $/ \mathrm{m} /$ is harder to isolate at the end of the sound because the $/ \mathrm{m} /$ easily transits into the next sound. The effect of the next sound of the word was analysed, but it showed no significant influence on the COG, so the sounds could be averaged.

The reason for analysing the parameter $\mathrm{COG}$ of each sound and not to look at other characteristics within the speech recordings was that the $\mathrm{COG}$ is an objective parameter, that can be acoustically analysed with the program PRAAT. The COG is a measurement that represents the average height of spectral frequencies. It is the most valuable characteristic of a sound [14]. Speech characteristics are variable within the same person [20], and it is possible to investigate speech with qualitative evaluation (an expert listening and assessing) but that remains subjective. Since there is little to no knowledge about the speech characteristics after a full rehabilitation of tooth wear, the objective analysis using COG was chosen as a starting point.

The patients' subjective opinion about their own speech, quality was evaluated using the SHI, showing very low means. This questionnaire may not have focused enough on tooth wear to evaluate the subtle changes in patients with a full rehabilitation due to tooth wear, resulting in no significant differences after the adaptation period of 1 month compared to the baseline $\left(T_{0}\right)$ registration, $p=0.054$. Only the subscale functional showed a significant improvement of 0.39 scale point.

Looking deeper into the different questions, three questions stood out for this specific study group, namely question P3, F3 and F4. Before treatment, many patients pointed out these problems to their dentist, even before filling in the SHI. So overall, the main speech problems in daily life for these patients were represented in the questionnaire. 
Table 3 Mean overall and subscale SHI-scores for $T_{0}$ (before treatment) and $T_{2}$ (1 month after treatment), and results of paired $t$ tests to evaluate the changes over time. $T_{0}$ was compared with $T_{2}$ to evaluate the impact of the treatment after adaptation

\begin{tabular}{lllll}
\hline SHI questionnaire & Mean $T_{0}( \pm \mathrm{SD})$ & Mean $T_{2}( \pm \mathrm{SD})$ & Mean diff. $T_{0}-T_{2}$ & $95 \%$ CI of diff. \\
\hline Total overall score & $0.52( \pm 0.45)$ & $0.29( \pm 0.24)$ & 0.23 & {$[0.00 \ldots 0.47]$} \\
Subscale physical & $0.84( \pm 0.63)$ & $0.59( \pm 0.44)$ & 0.25 & 0.054 \\
Subscale functional & $0.58( \pm 0.48)$ & $0.19( \pm 0.24)$ & 0.39 & 0.160 \\
Subscale emotional & $0.14( \pm 0.34)$ & $0.08( \pm 0.25)$ & 0.06 & {$[0.11 \ldots 0.60]$} \\
\hline
\end{tabular}

To assess the effect of the dental changes during treatment, the applied VDO in $\mathrm{mm}$ and the lengthening of the upper central incisors in mm were introduced in a regression model of COG changes. The applied VDO had no significant impact on any of the sounds and was therefore left out of the analysis. This study showed that the mean COG for the sounds $/ \mathrm{s} /, / \mathrm{t} /$, $/ \mathrm{d} /, / \mathrm{f} /, / \mathrm{v} /$ and $/ \mathrm{m} /$ decreased due to the treatment, when taking into account the mean change in the length of the central

Table 4 Results of the statistical analysis of the COG-changes for the analysed sounds. For the difference between $T_{0}$ and $T_{1}$, the intercept of the regression models shows the change in COG (at the centered mean increase of the length of the incisors: $2.6 \mathrm{~mm}$ ). The effect of the actual extent of change in incisor length (factor $\Delta$ length incisors) varied between the sounds. For the difference between $T_{1}$ and $T_{2}$, the intercept of the regression models is the change in COG in the situation where the change incisors. For the sounds /t/ and /f/, the degree of lengthening of the incisors significantly influenced the treatment effect.

The effect on these sounds may be explained by their formation: the / $\mathrm{f} /$ is produced through a light contact between the inside of the lower lip and the upper front teeth, while the $/ t /$ is produced with the tip of the tongue touching the roof of the mouth near or against the front teeth [21]. This may have led to a substantial effect on COG. The absence of a significant

in COG between $T_{0}$ and $T_{1}$ was 0 . This intercept does not have a natural interpretation. The relevant information is contained in the factor $\Delta \mathrm{COG}$ $T_{1}-T_{0}$, which represents the effect of the initial (or treatment) change (between $T_{0}$ and $T_{1}$ ) on the change between $T_{1}$ and $T_{2}$ (or adaptation). The ICC explained the clustering of repeated sounds (within one moment $\left(T_{0} / T_{1}\right.$ or $\left.T_{2}\right)$ within the patient. ICC $=0$, no clustering, ICC $=1$, distribution between patients, not within the patients themselves

\begin{tabular}{|c|c|c|c|c|c|c|c|}
\hline Sound & $T_{0} \mathrm{COG}(\mathrm{Hz}) \pm \mathrm{sd}$ & Moment & $\mathrm{ICC}$ & & Effect $(\mathrm{Hz})$ & $95 \%$ CI of diff. & $p$ value \\
\hline$/ \mathrm{s} /$ & & $T_{0} \rightarrow T_{1}$ & 0.061 & Intercept & -968 & {$[-1332 \ldots-598]$} & $<0.001$ \\
\hline \multirow[t]{3}{*}{$N=83$} & \multirow[t]{3}{*}{$5628 \pm 1483$} & & & $\Delta$ length incisors & -7.31 & {$\left[\begin{array}{llll}-322 & \ldots & 309\end{array}\right]$} & 0.967 \\
\hline & & $T_{1} \rightarrow T_{2}$ & 0.579 & Intercept & -515 & {$[-974 \ldots-58.6]$} & 0.027 \\
\hline & & & & $\Delta \mathrm{COG} T_{1}-T_{0}$ & -0.34 & {$[-0.46 \ldots-0.23]$} & $<0.001$ \\
\hline$/ t /$ & \multirow{4}{*}{$2952 \pm 2304$} & $T_{0} \rightarrow T_{1}$ & 0.037 & Intercept & -372 & {$[-814 \ldots 63]$} & 0.093 \\
\hline \multirow[t]{3}{*}{$N=83$} & & & & $\Delta$ length incisors & 502 & {$[124-878]$} & 0.009 \\
\hline & & $T_{1} \rightarrow T_{2}$ & 0.181 & Intercept & -130 & {$\left[\begin{array}{llll}-574 & \ldots & 303\end{array}\right]$} & 0.545 \\
\hline & & & & $\Delta \mathrm{COG} T_{1}-T_{0}$ & -0.29 & {$[-0.46 . .-0.11]$} & $<0.001$ \\
\hline$/ \mathrm{d} /$ & \multirow{4}{*}{$747 \pm 1013$} & $T_{0} \rightarrow T_{1}$ & 0.357 & Intercept & -44.6 & {$\left[\begin{array}{lll}-370 & \ldots & 281\end{array}\right]$} & 0.788 \\
\hline \multirow[t]{3}{*}{$N=82$} & & & & $\Delta$ length incisors & 138 & {$\left[\begin{array}{lll}-142 & \ldots 419\end{array}\right]$} & 0.333 \\
\hline & & $T_{1} \rightarrow T_{2}$ & 0 & Intercept & -40.3 & {$\left[\begin{array}{llll}-150 & \ldots & 69\end{array}\right]$} & 0.471 \\
\hline & & & & $\Delta \mathrm{COG} T_{1}-T_{0}$ & -0.32 & {$[0.44 \ldots-0.21]$} & $<0.001$ \\
\hline$/ \mathrm{f} /$ & \multirow{4}{*}{$6607 \pm 2154$} & $T_{0} \rightarrow T_{1}$ & 0.176 & Intercept & -1075 & {$[-1736 \ldots-409]$} & 0.001 \\
\hline \multirow[t]{3}{*}{$N=66$} & & & & $\Delta$ length incisors & 767 & {$\left[\begin{array}{llll}198.3 & \ldots & 1338\end{array}\right]$} & 0.008 \\
\hline & & $T_{1} \rightarrow T_{2}$ & 0.374 & Intercept & 375 & {$\left[\begin{array}{llll}-799 & \ldots & 587\end{array}\right]$} & 0.532 \\
\hline & & & & $\Delta \mathrm{COG} T_{1}-T_{0}$ & -0.55 & {$[-0.85 \ldots-0.24]$} & $<0.001$ \\
\hline$/ \mathrm{v} /$ & \multirow{4}{*}{$4019 \pm 2747$} & $\mathrm{~T}_{0} \rightarrow \mathrm{T}_{1}$ & 0.091 & Intercept & -361 & {$\left[\begin{array}{llll}-1029 & \ldots & 311\end{array}\right]$} & 0.294 \\
\hline \multirow[t]{3}{*}{$N=83$} & & & & $\Delta$ length incisors & 380 & [195 ... 959] & 0.194 \\
\hline & & $T_{1} \rightarrow T_{2}$ & 0.101 & Intercept & -399 & {$\left[\begin{array}{llll}-985 & \ldots & 185\end{array}\right]$} & 0.179 \\
\hline & & & & $\Delta \mathrm{COG} T_{1}-T_{0}$ & -0.45 & {$[-0.64 \ldots-0.27]$} & $<0.001$ \\
\hline$/ \mathrm{m} /$ & \multirow{4}{*}{$229 \pm 36$} & $T_{0} \rightarrow T_{1}$ & 0.065 & Intercept & -0.19 & {$\left[\begin{array}{llll}-12.3 & \ldots & 11.9\end{array}\right]$} & 0.970 \\
\hline \multirow[t]{3}{*}{$N=84$} & & & & $\Delta$ length incisors & 0.87 & {$\left[\begin{array}{llll}-9.82 & \ldots & 11.5\end{array}\right]$} & 0.877 \\
\hline & & $T_{1} \rightarrow T_{2}$ & 0.059 & Intercept & 16.0 & {$\left[\begin{array}{lll}4.57 & . .27 .5\end{array}\right]$} & 0.006 \\
\hline & & & & $\Delta \mathrm{COG} T_{1}-T_{0}$ & -0.68 & {$[-0.91 \ldots-0.48]$} & $<0.001$ \\
\hline
\end{tabular}


Ivl

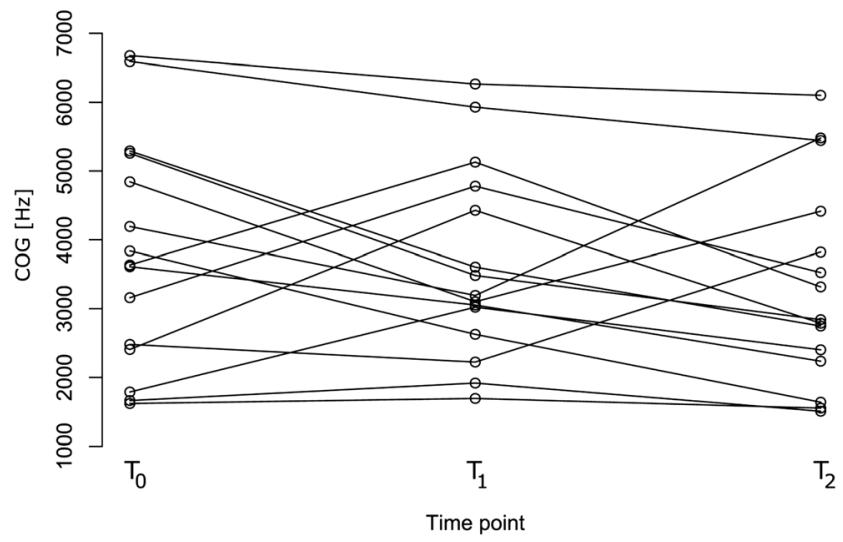

Fig. 1 Graph of the COG of the sound /v/ at the three different measurement moments for each patient (data points linked by lines per patient). The rebound effect may be observed for most patients, both when the initial change was positive and when it was negative

effect for the sounds $/ \mathrm{v} /$ and $/ \mathrm{d} /$ may also be found in the articulatory information, as the $/ \mathrm{v} /$ and $/ \mathrm{d} /$ are voiced sounds, unlike the /t/ and /f/ which are voiceless. Looking at the results of the SHI questionnaire, this pronounced effect did not lead to a negative impact on speech perception by the patient.

We expected that the increase of length of incisor teeth would have an effect on the /s/ sound; however, this was not observed. The impact of the whole treatment did show a significant impact, $p<0.001$, for the sound /s/. The verbal complaints of patients of a temporarily feeling of lisping, which was over within a few weeks, cannot be explained with the increase in length of the incisors. It is possible that for the $/ \mathrm{s} /$, the thickness on palatal side of the incisors may have had a greater effect, causing the temporarily feeling of lisping. The $/ \mathrm{s} /$ is created involving two constrictions: the narrow channel between the tongue and the palate or alveolar ridge, and the second constriction is formed by the teeth, against which the airflow is directed.

The COG after 1 month of adaptation showed a rebound effect, dependent on the initial treatment change in COG $T_{0^{-}}$ $T_{1}$. Interestingly, the rebound occurred irrespective of the direction, positive or negative, of the treatment effect.

The clinical relevance of this finding is not yet explained, but it may be a positive sign that the impact of a full rehabilitation does not result in lasting and possibly detrimental changes in the COG of the investigated sounds.

It is difficult to compare the COG values in this study to a 'golden standard' for speech. There is a lot of variations possible in the pronunciation, and dialects may have an influence [21]. On the website www.taalportaal.org, the Dutch language is investigated covering syntax, morphology and phonology. For the different investigated sounds: /s/, /f/, /v/, /d/, /t/ and /m/ , there are indications in which region the $\mathrm{COG}$ in $\mathrm{Hz}$ may be located [21]. These values strongly depend on the way they are obtained for example quality of the recordings, or amount of background noise that had to be cancelled out with a filter. Therefore, absolute COG values should probably not be compared across studies. Comparisons with other studies in the field of dentistry are also difficult because of methodological differences and the very limited literature on speech in relation to dentistry. But in the case of our study, the exact interpretation of the $\mathrm{COG}$ value (i.e. low or high $\mathrm{COG}$ value) is not important because the parameter COG was only used to detect speech changes within the same individual during repeated measurements in the course of time.

Further investigation may entail more recording moments, different parameters such as listening experiments or questionnaires specific designed for patients with tooth wear to come to a conclusion about the clinical implication of a full rehabilitation on speech characteristics.

\section{Conclusion}

Acoustic speech characteristics of tooth wear patients tend to change after full composite rehabilitation with a rebound effect after 1 month of adaptation, dependent on the size of the initial change right after treatment completion. Tooth wear patients perceive a subjective improvement in speech function after treatment.

Acknowledgements We would like to thank Joop Kerkhoff for his help and education about the program PRAAT. We also would like to thank Annemarie Schokkin for her help with analysing all speech samples.

\section{Compliance with ethical standards}

Conflict of interest The authors declare that they have no conflict of interest.

Ethical approval All procedures performed in studies involving human participants were in accordance with the ethical standards of the institutional and/or national research committee and with the 1964 Helsinki declaration and its later amendments or comparable ethical standards.

Informed consent Consent was obtained from all individual participants included in the study.

Open Access This article is licensed under a Creative Commons Attribution 4.0 International License, which permits use, sharing, adaptation, distribution and reproduction in any medium or format, as long as you give appropriate credit to the original author(s) and the source, provide a link to the Creative Commons licence, and indicate if changes were made. The images or other third party material in this article are included in the article's Creative Commons licence, unless indicated otherwise in a credit line to the material. If material is not included in the article's Creative Commons licence and your intended use is not permitted by statutory regulation or exceeds the permitted use, you will need to obtain permission directly from the copyright holder. To view a copy of this licence, visit http://creativecommons.org/licenses/by/4.0/. 


\section{References}

1. Lussi A, Carvalho TS (2014) Erosive tooth wear: a multifactorial condition of growing concern and increasing knowledge. Monogr Oral Sci 25:1-15

2. Carvalho TS, Lussi A, Jaeggi T, Gambon DL (2014) Erosive tooth wear in children. Monogr Oral Sci 25:262-278

3. Randall RC, Vrijhoef MM, Wilson NH (2002) Current trends in restorative dentistry in the UK: a Delphi approach. J Dent 30(4): $177-187$

4. Loomans B, Opdam N (2018) A guide to managing tooth wear: the Radboud philosophy. Br Dent J 224(5):348-356

5. Mehta SB, Banerji S, Millar BJ, Suarez-Feito JM (2012) Current concepts on the management of tooth wear: part 4 . An overview of the restorative techniques and dental materials commonly applied for the management of tooth wear. Br Dent J 212(4):169-177

6. Mehta SB, Banerji S, Millar BJ, Suarez-Feito JM (2012) Current concepts on the management of tooth wear: part 1. Assessment, treatment planning and strategies for the prevention and the passive management of tooth wear. Br Dent J 212(1):17-27

7. The glossary of prosthodontic terms, J Prosthet Dent 94(1) (2005) 10-92

8. Miles TS, Nauntofte B, Svensson P (2004) Clinical oral physiology. Quintessence Publishing Co., Copenhagen

9. Pena-Brooks A, Hegde M (2000) Assessment and treatment of articulation \& phonological disorders in children. Pro-ed, Texas (TX)

10. Hammond RJ, Beder OE (1984) Increased vertical dimension and speech articulation errors. J Prosthet Dent 52(3):401-406

11. Burnett CA, Clifford TJ (1999) The mandibular speech envelope in subjects with and without incisal tooth wear. Int J Prosthodont 12(6):514-518
12. Ishigaki S, Nakamura T, Akanishi M, Maruyama T (1989) Clinical classification of maximal opening and closing movements. Int $\mathrm{J}$ Prosthodont 2(2): 148-154

13. Sterenborg B, Kalaykova SI, Loomans BAC, Huysmans M (2018) Impact of tooth wear on masticatory performance. J Dent

14. Boersma P (2001) Praat, a system for doing phonetics by computer. Glot International 5(9/10):341-345

15. L. Van den Steen, G. Nuffelen, C. Guns, M. De Groote, L. Pinson, M. Bodt, De Spraak Handicap Index: Een instrument voor zelfevaluatie by dysartriepatienten, 2011

16. Wetselaar P, Lobbezoo F, Koutris M, Visscher CM, Naeije M (2009) Reliability of an occlusal and nonocclusal tooth wear grading system: clinical use versus dental cast assessment. Int $\mathrm{J}$ Prosthodont 22(4):388-390

17. Morley J, Eubank J (2001) Macroesthetic elements of smile design. J Am Dent Assoc 132(1):39-45

18. Knuijt S, Kalf JG, van Engelen BGM, de Swart BJM, Geurts ACH (2017) The Radboud dysarthria assessment: development and clinimetric evaluation. Folia Phoniatr Logop 69:143-153

19. Rietveld ACM, van Heuven VJ (2016) Algemene fonetiek. Coutinho, Bussum

20. Wolfe V, Cornell R, Fitch J (1995) Sentence/vowel correlation in the evaluation of dysphonia. J Voice 9(3):297-303

21. F. Landsbergen, C. Tiberius, R. Dernison, Taalportaal: an Online Grammar of Dutch and Frisian, Proceedings of the Ninth International Conference on Language Resources and Evaluation LREC'14 (2014) 2206-2210

Publisher's note Springer Nature remains neutral with regard to jurisdictional claims in published maps and institutional affiliations. 